OPEN ACCESS

Edited by:

Patrick Ho Yu Chung, University of Hong Kong,

Hong Kong SAR, China

Reviewed by:

Emmanuelle Seguier,

Schneider Children's Medical

Center, Israe

Michele ILARI,

"G. Salesi" Children's Hospital, Italy

Vicky Wong,

Prince of Wales Hospital, China

*Correspondence:

Xueqiang Yan yanxueqiang1@163.com

Specialty section:

This article was submitted to

Pediatric Surgery

a section of the journal

Frontiers in Pediatrics

Received: 23 November 2021 Accepted: 20 December 2021

Published: 28 January 2022

Citation:

Yuan Q, Duan X and Yan X (2022) Primary Omental Lipoma in a Child: $A$ Case Report and Literature Review.

Front. Pediatr. 9:820845.

doi: 10.3389/fped.2021.820845

\section{Primary Omental Lipoma in a Child: A Case Report and Literature Review}

\author{
Qiang Yuan, Xufei Duan and Xueqiang Yan*
}

Department of Pediatric Surgery, Wuhan Children's Hospital (Wuhan Maternal and Child Healthcare Hospital), Tongji Medical College, Huazhong University of Science and Technology, Wuhan, China

Background: Lipoma is a common benign tumor derived from adipose tissue, with an incidence of nearly $10 \%$. It is the most common mesenchymal tumor throughout the body. However, the pathogenesis of lipoma is not clear yet, and the increased incidence is attributable to obesity, elevated serum cholesterol, diabetes, trauma, radiation, familial predisposition, and chromosome. Primary omental tumor is a rare lipoma occurring in the greater omentum, most of which is reported in the form of clinical case reports. Nevertheless, primary omental tumor is even rarer in children. To date, there have been few reports of clinical cases.

Case Presentation: We report a rare case of primary omental lipoma in a 6-year-old boy. After an accidental fall, a CT scan found that he had a tumor in the left upper abdomen. He had no history of abdominal pain, abdominal mass, vomiting, etc. The boy was admitted to the hospital within 3 days, and was diagnosed with an intraabdominal tumor. After admission, abdominal ultrasound and enhanced CT showed a $71 \times 40 \times 60 \mathrm{~mm}$ mass in the left middle abdomen, which was considered a lipoma. There was no abnormality in tumor markers. Through laparoscopic surgery, intraoperative exploration revealed that the tumor was located in the left mid-upper abdomen, and was yellow, solid, soft, and isolated. The intraoperative diagnosis was an omental lipoma. We used an ultrasonic knife to resect the omentum close to the base of the tumor. The tumor was completely resected, put in a retrieval bag and sealed. Finally, the left and right sides of the umbilical incision were extended to take out the tumor tissue. The child received liquid food $6 \mathrm{~h}$ after the operation and was discharged 3 days later. The postoperative pathological diagnosis was an omental lipoma. He was seen at follow-up 3 months after discharge and had no complaints, an abnominal ultrasound showed no tumor recurrence.

Conclusion: Primary omental lipoma in children is a rare benign tumor of the omentum. Its etiology and pathology are not clear. US, CT, and MRI can facilitate clinical diagnosis and preoperative evaluation. Laparoscopic surgery is an effective treatment, and the prognosis of children is favorable. This case is beneficial to improve the clinical knowledge of pediatric surgeons about this rare disease.

Keywords: primary omental lipoma, laparoscopy, child, US, resect 


\section{INTRODUCTION}

Primary omental lipoma is a rare benign tumor of the omentum. The pathogenesis is not clear, and there is no statistical data on its incidence. This lipoma in children is even rarer, most of which are reported in the form of clinical case reports. This study reports the diagnosis and treatment of a 6-year-old boy with primary omental lipoma. Meanwhile, it discusses the clinical characteristics as well as methods of diagnosis and treatment of primary omental lipoma based on previous literature data. This study was approved by the Research and Ethics Committee of our institution, and written informed consent was obtained from the patient's family.

\section{CASE PRESENTATION}

A 6-year-old male child was found to have a tumor in the left mid-upper abdomen on a CT examination of his abdomen after an accidental fall. The boy had no history of abdominal pain, abdominal mass, and vomiting. He was admitted to the hospital with an abdominal tumor within 3 days. The initial diagnosis was an intraperitoneal tumor. After admission, laboratory tests showed no abnormality in blood cell count, biochemical analysis, and tumor markers. Abdominal ultrasound and enhanced CT showed a mass in the left middle abdomen, which size was 71 $\times 40 \times 60 \mathrm{~mm}$. Intra-abdominal lipoma should be considered, and the blood-supplying vessels were derived from branches of the omental artery (Figures 1A,B).

We performed laparoscopy surgery on the child: three incisions for $5 \mathrm{~mm}$ trocars were made on the insufflated abdomen respectively at the left and right sides of the umbilicus and at the anti-Mc Burney's point. Intraoperative laparoscopic exploration revealed a tumor in the left part of middle and upper abdomen, with a size of about $70 \times 60 \times 50 \mathrm{~mm}$. It was yellow, solid, soft, isolated, and its base was derived from the greater omentum without torsion (Figure 2A). Preoperative and intraoperative assessments revealed that the tumor could be successfully and completely resected by laparoscopic surgery. The ultrasound
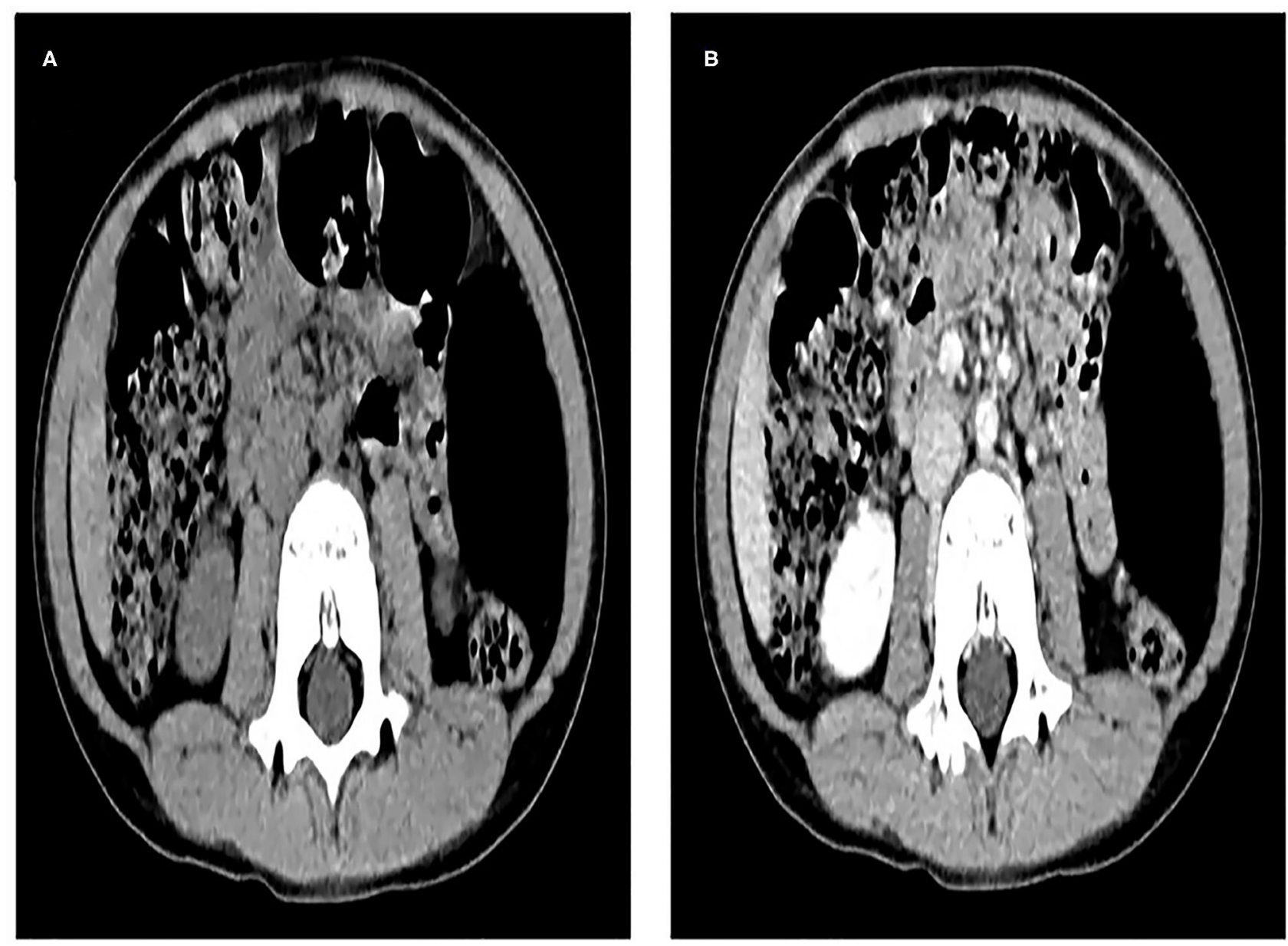

FIGURE 1 | (A), Computed tomography (CT) scan shows an elliptical, very low-density mass in the left middle abdomen, which is wrapped in the left abdominal cavity, and the surrounding intestine is slightly compressed and displaced. (B), The tumor was not enhanced on enhanced CT scan, and its blood supply arteries were not shown. 

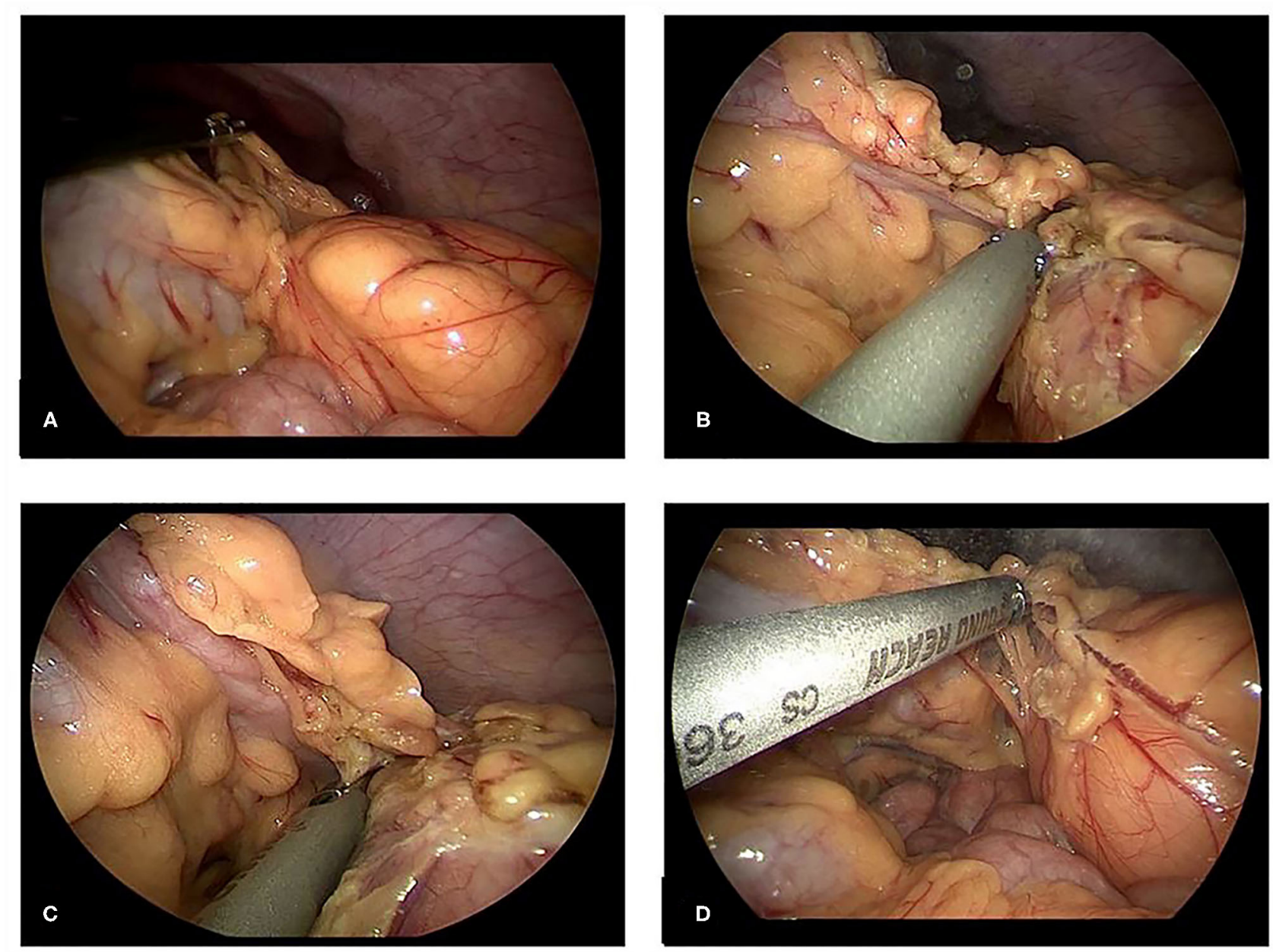

FIGURE 2 | (A), Laparoscopic examination of the left mid-upper abdominal tumor, which was yellow, solid, isolated, derived from the greater omentum, without torsion. (B,C), Ultrasonic knife separates the omentum and the tumor. (D), Ultrasonic scalpel coagulates the tumor blood supply artery.

knife cut off the greater omentum and the blood-supplying vessels at the base of the tumor. The tumor was completely removed and was placed in the specimen retrieval bag which was then closed. The ultrasound knife coagulated the residual end of the greater omentum to stop bleeding. No other abnormality was found in the abdominal cavity below the umbilicus to take out the tumor tissue completely (Figures 2B-D).

The operation time was $27 \mathrm{~min}$. The weight of the excised tumor tissue was $295 \mathrm{~g}$, size of about $80 \times 55 \times 30 \mathrm{~mm}$ with; the tumor capsule intact; and the cut surface is grayish-yellow and soft (Figure 3A). Microscopic examination showed that the tumor was composed of mature adipocytes without atypia (Figure 3B); and the diagnosis was of a benign omental lipoma. The patient was discharged 3 days postoperatively without further complication, follow-up abdominal ultrasonography after 3 months showed no recurrence of lipoma.

Pubmed, Springer Link, CNKI, and Wanfang databases were searched before June 2021 to retrieve the related literature of omental lipoma, a total of 12 clinical reports of primary omental lipoma in children were retrieved in the previous literature (Table 1).

\section{DISCUSSION AND CONCLUSIONS}

Lipoma is a common benign tumor derived from adipose tissue, with an incidence of nearly $10 \%$ (1). It is the most common mesenchymal tumor throughout the body. However, the pathogenesis of lipoma is not clear yet, and the increased incidence is attributable to obesity, elevated serum cholesterol, diabetes, trauma, radiation, familial predisposition, and chromosome abnormality $(2,3)$. The omentum is a double-layer membrane composed of peritoneum and adipose tissue, which is attached to the greater curvature of the stomach and transverse colon. It covers the abdominal organs in the abdominal cavity in the shape of a skirt, including blood vessels, nerves, lymphatic vessels, and connective tissue $(4,5)$. Primary omental lipoma is very rare, and most of which is described in the form of case reports. 

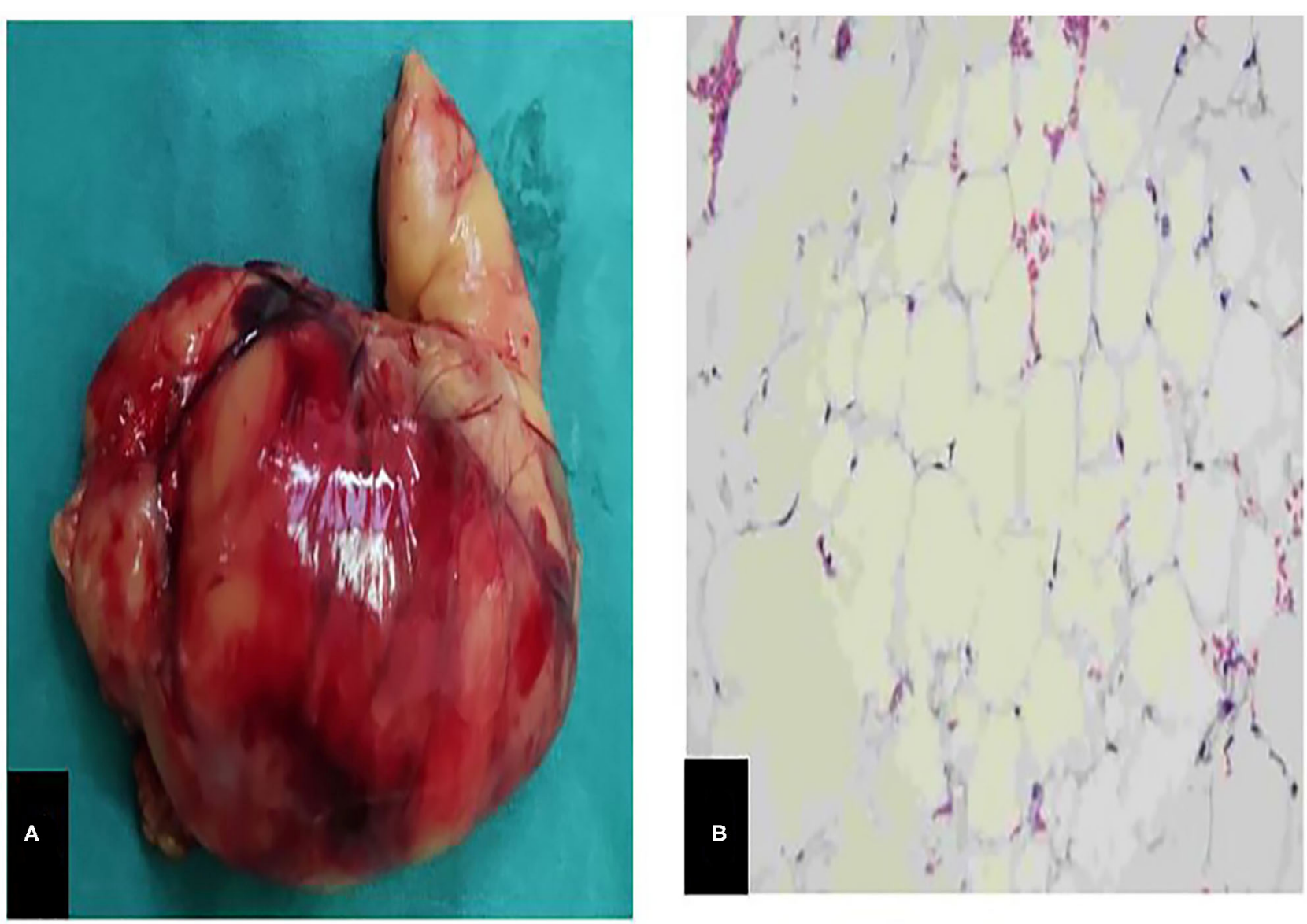

FIGURE 3 | (A), A piece of yellow tissue can be seen with the naked eye, $295 \mathrm{~g}$ in weight, about $80 \mathrm{~mm}$ *55mm * $30 \mathrm{~mm}$ in size, with intact capsule, solid and soft. (B), Microscopically, the tumor is composed of mature adipocytes without atypia.

TABLE 1 | Cases of children reported to have omental lipoma found in the available literature.

\begin{tabular}{|c|c|c|c|c|c|c|c|c|}
\hline Case & Authors & Year & Age & Sex & Clinical presentation & Weigh (g) & Size (cm) & Treatment \\
\hline 1 & Haller & 1978 & 3y & $\mathrm{F}$ & NR & NR & $8^{\star} 5^{\star} 4$ & Excision \\
\hline 2 & Giubilei & 1980 & $8 y$ & M & NR & NR & $18^{\star} 15^{\star} 8$ & Excision \\
\hline 4 & Barauskas & 2004 & $8 y$ & $\mathrm{~F}$ & NR & 720 & $11^{\star} 10^{\star} 8$ & Excision \\
\hline 5 & Luo & 2005 & $11 \mathrm{~m}$ & M & Abdominal distention & 1820 & $21^{\star} 15^{\star} 12$ & Excision \\
\hline 8 & Chaudhary & 2011 & $2 y$ & M & NR & NR & NR & Excision \\
\hline 9 & Cascini & 2012 & $19 m$ & M & Abdominal distention & 1185 & $22^{\star} 18^{\star} 8$ & Excision \\
\hline 10 & Cascini & 2012 & $7 y$ & $\mathrm{~F}$ & Abdominal distention & 2070 & $25^{\star} 22^{\star} 10$ & Excision \\
\hline 11 & Cascini & 2012 & $10 y$ & $\mathrm{~F}$ & Abdominal pain & 1370 & $18^{*} 12^{*} 10$ & Excision \\
\hline 12 & Kinjo & 2014 & $5 y$ & $\mathrm{~F}$ & NR & 335 & $8^{\star} 6 * 3$ & Excision \\
\hline
\end{tabular}

$N R$, not reported; $y$, years; $m$, moths.

A variety of pathologies have been reported in the clinic, such as leiomyosarcoma, fibrosarcoma, hemangiopericytoma, liposarcoma, leiomyoma, lipoma, fibroma, and mesothelioma tumors. Lipoma may be the rarest of all the above forms, with an extremely low incidence, accounting for about $7-9 \%$ of omental tumors $(6,7)$. 
Review-of the retrieved literature of 12 cases of primary omental lipoma in children, found that children with omental lipoma are usually asymptomatic and are discovered incidentally during medical examination, trauma, or abdominal diseases. The main symptoms of these children include abdominal pain, abdominal distention, and abdominal mass. Nausea, weight loss, and intraperitoneal hemorrhage occurred occasionally, and a few children were admitted to the emergency department due to torsion of the omentum. It has been reported that adult patients were hospitalized due to intussusception and intestinal obstruction $(8,9)$.

Common imaging methods for diagnosing omental tumors include ultrasound, CT, and MRI. It is clinically difficult to discriminate benign and malignant omental tumors. The differential diagnosis of omental lipoma includes lymphangioma, lymphoma, duplication of the digestive tract, and neuroblastoma. However, the main differential diagnosis is lipoblastoma (10). Omental lipoma is easily found by ultrasound examination. Ultrasound can show that the tumor has heterogeneous echo and abundant blood flow signals, it also reveals the size of the tumor. Smaller omental lipomas are sometimes misdiagnosed as normal mesenteric fat (11). Ultrasound elastography is a non-invasive imaging technology based on different tissue hardness which have been developed in recent years. It can measure the elasticity of the tissue, according to the various performance of the tissue under different external pressure, then to distinguish benign and malignant lesions. Zhang et al. (12) explored whether ultrasound elastography is effective in the diagnosis of benign and malignant omentum thickening. The results showed that the elasticity score of the malignant omentum thickening group was higher than that of the benign omentum thickening group $(P<0.01)$. In addition, ultrasoundguided percutaneous needle biopsy has been a commonly used method for the diagnosis of intra-abdominal lesions, such as liver, kidney, pancreas lesions, and other solid organs, but it is not commonly used for peritoneal and omental lesions. It may cause controversy by seeding tumor cells along the needle path (13). CT examination can show clear, uniform, and lowdensity intra-abdominal mass, which may have fibrous partitions and a few calcification points inside. Enhanced CT helps to assess the relationship between the tumor and surrounding organs, and also helps to identify the blood-supplying arteries of the tumor (14). MRI has high specificity for the diagnosis of simple lipoma and can distinguish it from well-differentiated liposarcoma. By using an MRI pulse sequence, lipomas show signal intensity similar to that of fat on high T1 signal and intermediate T2 signal. If there are thicker intervals inside the tumor, nodules, and non-fat-like masses, the proportion of fat in the lesion will decrease. It suggests the diagnosis of liposarcoma $(15,16)$.

\section{REFERENCES}

1. Sanchez MR, Golomb FM, Moy JA, Potozkin JR. Giant lipoma: case report and review of the literature. $J$ Am Acad Dermatol. (1993) 28(2 Pt 1$): 266-8$. doi: 10.1016/S0190-9622(08) 81151-6
Laparoscopic exploration is an important method to confirm that lipomas originate from the greater omentum during the operation, and surgical resection is an effective treatment for the lipoma of the greater omentum. The tumor can be completely resected under laparoscopy. When a patient is admitted to the hospital due to torsion of the greater omentum, laparoscopic exploration can be performed in the emergency department, both laparoscopic tumor resection and the greater omentum resection can be performed at the same time. According to previous reports in the literature, the recurrence rate after resection is $<5 \%$, mainly due to incomplete resection (17).

In conclusion, we reported a rare case of primary omental lipoma in children. By reviewing the previous literature, we found that primary omental lipoma in children is very rare. Preoperative ultrasound and enhanced CT can determine intraabdominal lipoma, which can also help to identify the origin of the tumor's blood vessels. Intraoperative exploration of laparoscopic surgery can confirm that the lipoma originates from the greater omentum. The lipoma can be completely resected under laparoscopic surgery. If combined with torsion of the greater omentum, partial resection of the greater omentum can be performed at the same time. Laparoscopic surgery is minimally invasive and generally without complications. The prognosis of the child is good.

\section{DATA AVAILABILITY STATEMENT}

The original contributions presented in the study are included in the article/supplementary material, further inquiries can be directed to the corresponding author.

\section{ETHICS STATEMENT}

The studies involving human participants were reviewed and approved by Wuhan Children's Hospital (Wuhan Maternal and Child Healthcare Hospital), Tongji Medical College, Huazhong University of Science and Technology. The patients/participants provided their written informed consent to participate in this study. Written informed consent was obtained from the individual(s), and minor(s)' legal guardian/next of kin, for the publication of any potentially identifiable images or data included in this article.

\section{AUTHOR CONTRIBUTIONS}

QY and XY designed the study, collected the clinical data, performed the statistical analysis, participated in the operation, and drafted the manuscript. XD participated in the operation and revised the article. All authors read and approved the final manuscript.

2. Enzinger FM, Weiss SW. Soft Tissue Tumors. 3rd ed. St Louis (Mo): Mosby (1995). p. 384-405.

3. Weiss SW. Lipomatous tumors. Monogr Pathol. (1996) 38:207-39.

4. Koppe MJ, Nagtegaal ID, de Wilt JH, Ceelen WP. Recent insights into the pathophysiology of omental metastases. J Surg Oncol. (2014) 110:670-5. doi: 10.1002/jso.23681 
5. Krist LF, Koenen H, Calame W, van der Harten JJ, van der Linden JC, Eestermans IL, et al.Ontogeny of milky spots in the human greater omentum: an immunochemical study. Anat Rec. (1997) 249:399404. doi: 10.1002/(SICI)1097-0185(199711)249:3<399::AID-AR11>3.0. CO;2-J

6. Dixon AY, Reed JS, Dow N, Lee SH. Primary omental leiomyosarcoma masquerading as hemorrhagic ascites. Hum Pathol. (1984) 15:233-7. doi: 10.1016/S0046-8177(84)80185-9

7. Tsurumi H, Okada S, Koshino Y, Oyama M, Higaki H, Shimokawa K, et al. A case of leiomyoblastoma (epithelioid leiomyosarcoma) of the greater omentum. Gastroenterol Jpn. (1991) 26:370-5. doi: 10.1007/BF02781927

8. Pintor-Tortolero J, Martínez-Núñez S, Tallón-Aguilar L, Padillo-Ruiz FJ. Colonic intussusception caused by giant lipoma: a rare cause of bowel obstruction. Int J Colorectal Dis. (2020) 35:1973-7.

9. Livne PM, Zer M, Shmuter Z, Dintsman M. Acute intestinal obstruction caused by necrotic mesenteric lipoma-a case report. Am J Proctol Gastroenterol Colon Rectal Surg. (1981) 32:19-22.

10. Abubakar AM, Mayun AA, Pindiga UH, Chinda JY, Adamu SA. Giant omental lipoma in a 13-year-old adolescent girl. J Pediatr Surg. (2009) 44:2230-2. doi: 10.1016/j.jpedsurg.2009.09.007

11. Cha JM, Lee JI, Joo KR, Choe JW, Jung SW, Shin HP, et al. Giant mesenteric lipoma as an unusual cause of abdominal pain: a case report and a review of the literature. J Korean Med Sci. (2009) 24:333-6. doi: 10.3346/jkms.2009.24.2.333

12. Zhang Y, Wang X, Tao C, Que Y, Zhao W, Chen B. Ultrasound elastography for differentiating benign from malignant thickened greater omentum. Eur Radiol. (2016) 26:2337-43. doi: 10.1007/s00330-015-4037-0

13. Beattie GC, Irwin ST. Torsion of an omental lipoma presenting as an emergency. Int $J$ Clin Pract Suppl. (2005) 130-1. doi: 10.1111/j.1368-504X.2005.00457.x

14. Shiroshita H, Komori Y, Tajima M, Bandoh T, Arita T, Shiraishi N, et al. Laparoscopic examination and resection for giant lipoma of the omentum: a case report and review of related literature. Surg Laparosc Endosc Percutan Tech. (2009) 19:e217-20. doi: 10.1097/SLE.0b013e3181b 97edd

15. Hamidi H, Rasouly N, Khpalwak H, Malikzai MO, Faizi AR, Hoshang MM, et al. Childhood giant omental and mesenteric lipoma. Radiol Case Rep. (2016) 11:41-4. doi: 10.1016/j.radcr.2015.12.003

16. Tirukonda P, Wu S, Brar J, Ng KS, Mirsadraee S. Trans arterial embolization of spontaneous intra-abdominal haemorrhage from omental lipoma. Case Rep Radiol. (2018) 2018:2926143. doi: 10.1155/2018/29 26143

17. Squillaro AI, Chow MD, Arias F, Sadimin ET, Lee YH. A giant childhood mesenteric lipoblastoma with extensive maturation. Front Pediatr. (2020) 8:404. doi: $10.3389 /$ fped.2020.00404

Conflict of Interest: The authors declare that the research was conducted in the absence of any commercial or financial relationships that could be construed as a potential conflict of interest.

Publisher's Note: All claims expressed in this article are solely those of the authors and do not necessarily represent those of their affiliated organizations, or those of the publisher, the editors and the reviewers. Any product that may be evaluated in this article, or claim that may be made by its manufacturer, is not guaranteed or endorsed by the publisher.

Copyright $\odot 2022$ Yuan, Duan and Yan. This is an open-access article distributed under the terms of the Creative Commons Attribution License (CC BY). The use, distribution or reproduction in other forums is permitted, provided the original author(s) and the copyright owner(s) are credited and that the original publication in this journal is cited, in accordance with accepted academic practice. No use, distribution or reproduction is permitted which does not comply with these terms. 